\title{
Introducing edge-biregular maps
}

\author{
Olivia Reade ${ }^{1}$
}

Received: 6 December 2020 / Accepted: 23 October 2021 / Published online: 3 December 2021

(c) The Author(s) 2021

\begin{abstract}
We introduce the concept of alternate-edge-colourings for maps and study highly symmetric examples of such maps. Edge-biregular maps of type $(k, l)$ occur as smooth normal quotients of a particular index two subgroup of $T_{k, l}$, the full triangle group describing regular plane $(k, l)$-tessellations. The resulting colour-preserving automorphism groups can be generated by four involutions. We explore special cases when the usual four generators are not distinct involutions, with constructions relating these maps to fully regular maps. We classify edge-biregular maps when the supporting surface has non-negative Euler characteristic, and edge-biregular maps on arbitrary surfaces when the colour-preserving automorphism group is isomorphic to a dihedral group.
\end{abstract}

Keywords Symmetric map · Automorphism · Triangle group · Regular · 2-Orbit map

\section{Introduction}

A map is an embedding of a connected graph in a surface such that the image of the graph divides the surface into regions which we call faces, while the interior of each face is homeomorphic to an open disc. This paper addresses maps with alternateedge-colourings and introduces the algebraic theory underlying the most symmetric examples of such maps.

Section 2 introduces the concept of an alternate-edge-colouring for a map, a condition which is equivalent to the medial map being bipartite. We present the monodromy group for this type of map and relate it to the colour-preserving automorphism group. We then study some properties arising from the algebraic background, focussing on the subclass of these maps which have the largest possible colour-preserving automorphism group, maps which we call edge-biregular.

Olivia Reade

olivia.jeans@open.ac.uk

1 Open University, Milton Keynes MK7 6AA, UK 
Section 3 addresses special cases of edge-biregular maps, for example when there are semi-edges or boundary components, including constructions relating these to fully regular maps.

A classification of edge-biregular maps supported by surfaces of non-negative Euler characteristic is shown in Sect. 4.

Section 5 is devoted to a classification of edge-biregular maps on surfaces of negative Euler characteristic where the colour-preserving automorphism group is isomorphic to a dihedral group, while Sect. 6 contains concluding remarks.

\section{Preliminaries}

\subsection{Alternate-edge-colourings}

A map has an alternate-edge-colouring when it is possible to colour the edge set using two colours such that consecutive edges around any given face will be differently coloured and so also two consecutive edges in the cyclic order of edges around any vertex will be assigned different colours. This property is equivalent to the map having a bipartite medial graph. In our diagrams, we will use a bold line to denote an edge of one colour (which we call shaded) and dashed lines to indicate edges of the other colour (unshaded).

We note that a map having an alternate-edge-colouring is also equivalent to being able to define an orientation on the set of corners of a map such that adjacent corners have the opposite orientation. The orientations on the corners can then be defined to be consistent with sweeping the corners always in the same direction with respect to the colouring, for example from the shaded edge to the unshaded edge. In this last sense, it is a similar definition to the pseudo-orientable maps introduced by Wilson in [14], but we are assigning the orientations to corners of the map rather than vertices.

An example of a map with an assigned alternate-edge-colouring is shown in Fig. 1.

Fig. 1 A map on a sphere with an assigned alternate-edgecolouring

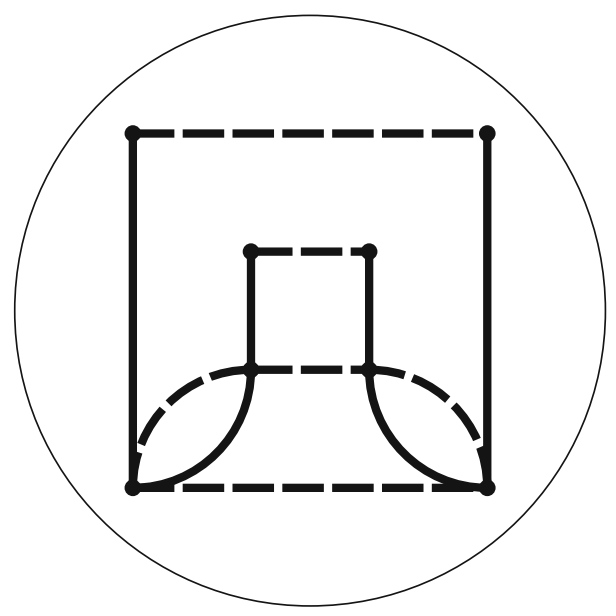




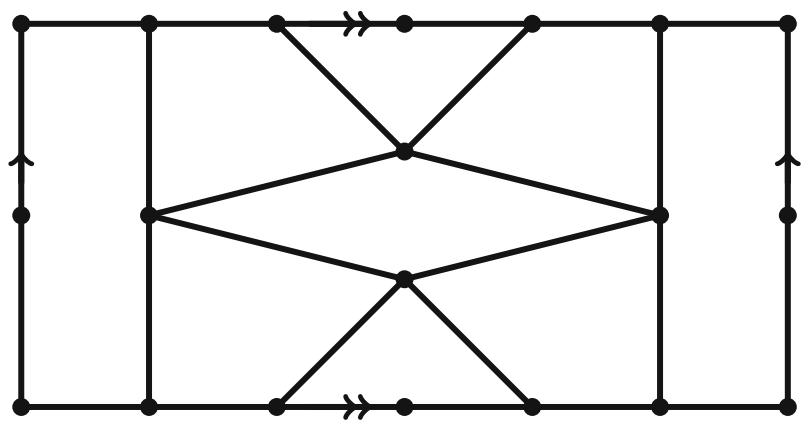

Fig. 2 A toroidal map (edges of the rectangle identified in the usual way) with no alternate-edge-colouring

In general, unless stated otherwise, we will assume we are working with maps supported by closed surfaces, that is, maps without boundary components. In this case, every vertex of a map with an alternate-edge-colouring must have even valency and all the faces will have even length closed boundary walks. However, having even face lengths and valencies is not sufficient for a map to have an alternate-edge-colouring. An example of a map which has only even length faces, and only even degree vertices, is shown in Fig. 2. Any attempt to form an alternate-edge-colouring will result in a contradiction. For example, the (single) edge with the double arrow would "want" to be coloured with both colours, which is clearly impossible. The same is true for the single arrow edge.

Both examples and non-examples of such maps exist on non-orientable surfaces too. If the left and right edges of the rectangle in Fig. 2 are identified in the opposite direction from each other, then we have a non-orientable map with even valency and even face length for which there is still no alternate-edge-colouring. However, the Klein bottle does support maps with alternate-edge-colourings, as we will see later, in Sect. 4.2.2.

\subsection{The corner-monodromy group}

We define flags of a map to be the faces of its barycentric subdivision. We refer to the vertices of the barycentric subdivision as labels. Each flag thus has three labels, one for each dimension 0, 1 and 2, respectively, corresponding to the vertex, edge and face of the map with which that flag is associated.

In a map with an assigned alternate-edge-colouring, that is a map which has been given an alternate-edge-colouring, each flag will inherit a natural colouring, shaded or unshaded, according to the colour of the edge with which it associated. The pair of flags which together form a corner (that is, the two neighbouring flags from one face which meet in a natural way at a corner of that face) will therefore have one flag of each colour, and the whole map can be decomposed into the set $\mathcal{C}$ of ready-coloured corners, that is corners with an assigned colouring.

The gluing instructions for the elements of the set $\mathcal{C}$ for a map with an assigned alternate-edge-colouring are in a sense the ready-coloured-corner-monodromy group 


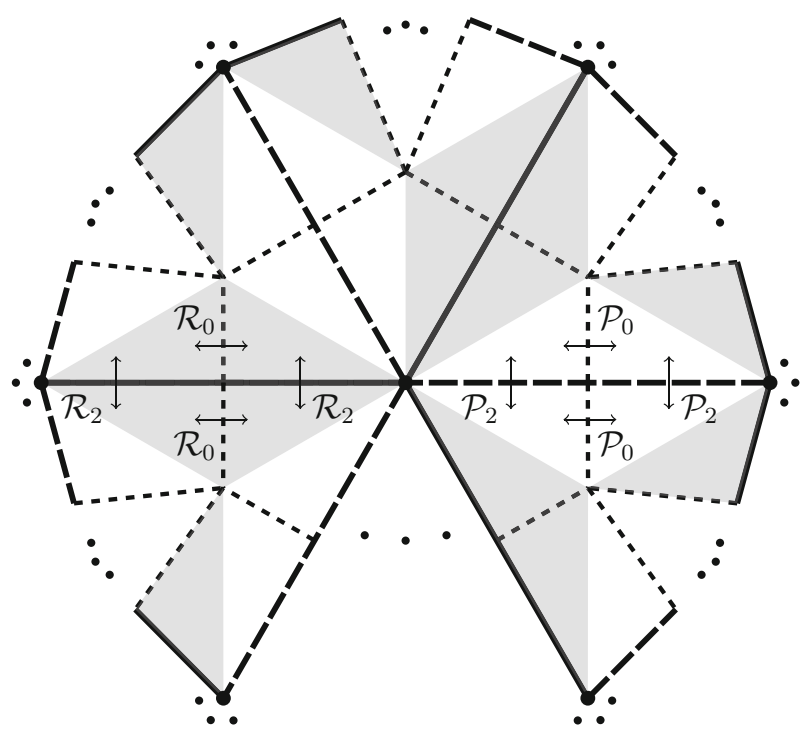

Fig. 3 The action of $\mathcal{R}_{0}, \mathcal{R}_{2}$ and $\mathcal{P}_{0}, \mathcal{P}_{2}$ on certain corners of a map with alternate-edge-colouring

$G$. The group $G$ acts on the right of $\mathcal{C}$ and is generated by four involutions as follows. Following well-established notation, see [9], we define the involutions $\mathcal{R}_{0}, \mathcal{R}_{2}$ to be the operations which swap every shaded flag with its neighbour, respectively, along and across a shaded edge. The subscripts in this notation indicate the dimension of the labels of the adjacent shaded flags which are interchanged by the involution. But the action of this group is on the set of two-coloured corners, not flags, so we see $\mathcal{R}_{0}, \mathcal{R}_{2}$ swaps every ready-coloured-corner with its unique neighbour, respectively, along and across a shaded edge, while we define $\mathcal{P}_{0}, \mathcal{P}_{2}$ to be the involutions which interchange every ready-coloured-corner with its unique neighbour, respectively, along and across an unshaded edge.

Figure 3 shows the action of $\mathcal{R}_{0}, \mathcal{R}_{2}, \mathcal{P}_{0}, \mathcal{P}_{2}$ on a selection of ready-colouredcorners. Each corner in the diagram is outlined by a bold line, a long-dashed line and two short-dashed lines, and consists of one flag of each colour. The two corners $c$ and $c \mathcal{R}_{0}$ are thus adjacent along a shaded edge, while $c$ and $c \mathcal{R}_{2}$ are adjacent across a shaded edge and $c$ is adjacent to $c \mathcal{P}_{0}$ and $c \mathcal{P}_{2}$, respectively, along and across an unshaded edge.

The group $G=\left\langle\mathcal{R}_{0}, \mathcal{R}_{2}, \mathcal{P}_{0}, \mathcal{P}_{2}\right\rangle$ for a map with an assigned alternate-edgecolouring which is decomposed into its ready-coloured-corners is then isomorphic to a quotient group of $\Gamma:=\left\langle\mathfrak{R}_{0}, \mathfrak{R}_{2}\right\rangle *\left\langle\mathfrak{P}_{0}, \mathfrak{P}_{2}\right\rangle \cong V_{4} * V_{4}$ defined by the natural epimorphism $\phi: \Gamma \rightarrow G$ where $\phi: \mathfrak{R}_{i} \rightarrow \mathcal{R}_{i}$ and $\phi: \mathfrak{P}_{i} \rightarrow \mathcal{P}_{i}$. The group $\Gamma$ is the ready-coloured-corner-monodromy group of a universal map for the class of alternateedge-colourable maps, from which any map with this property can be determined by the natural epimorphism $\phi$. 


\subsection{The colour-preserving automorphism group}

We consider the group $H$ of automorphisms acting on the (left of the) set $\mathcal{C}$ which preserve both the structure and the colouring of the map. By this, we mean all automorphisms which act such that the images of two neighbouring corners will share the same type of adjacency as their pre-images, that is along or across either a shaded or an unshaded edge. The group $H$ thus consists of all permutations of the set $\mathcal{C}$ which commute with all the gluing instructions in $G$. Hence, $H=\left\{h \in \operatorname{Sym}_{\mathcal{C}} \mid h(c) \mathcal{R}_{i}=\right.$ $h\left(c \mathcal{R}_{i}\right)$ and $h(c) \mathcal{P}_{i}=h\left(c \mathcal{P}_{i}\right)$ for all $\left.c \in \mathcal{C}, i \in\{0,2\}\right\}$, that is, $H$ is the centraliser of $G$ in the symmetric group acting on the set $\mathcal{C}$.

\subsection{Edge-biregular maps}

We will always assume that all our maps are connected, and hence, the structurepreserving condition for our map automorphisms forces the (left) action of $H$ on $\mathcal{C}$ to be semi-regular, that is fixed-point free. The largest possible automorphism group $H$ acting on $\mathcal{C}$, the set of (coloured) corners of a map with assigned alternate-edge-colouring, will be when $H$ acts transitively on the set $\mathcal{C}$. The action being both fixed-point free and transitive means the action is regular and this allows us to identify the elements of the group $H$ with the corners in the set $\mathcal{C}$. We mark an arbitrary corner $C$ which is labelled as the identity element and refer to the structures incident to this corner as distinguished. The automorphisms in $H$ are then generated by the involutions $\left\langle r_{0}, r_{2}, \rho_{0}, \rho_{2}\right\rangle$ which act locally as reflections in the boundaries of this marked corner, (respectively, along or across the distinguished shaded edge and along or across the distinguished unshaded edge), while preserving all the adjacency relationships between corners. See Fig. 4.

When $H$ acts regularly on the set $\mathcal{C}$ of corners of a map with an assigned alternateedge-colouring, we say the map is edge-biregular. Henceforth, except in some special cases, we will be considering edge-biregular maps with even valency $k$ and even face length $l$ which we describe with the canonical form $\left(H ; r_{0}, r_{2}, \rho_{0}, \rho_{2}\right)$ where the generators act as described above, and $H$ is a group with the partial presentation

$$
H=\left\langle r_{0}, r_{2}, \rho_{0}, \rho_{2} \mid r_{0}^{2}, r_{2}^{2}, \rho_{0}^{2}, \rho_{2}^{2},\left(r_{0} r_{2}\right)^{2},\left(\rho_{0} \rho_{2}\right)^{2},\left(r_{2} \rho_{2}\right)^{k / 2},\left(r_{0} \rho_{0}\right)^{l / 2}, \ldots\right\rangle .
$$

The exceptional cases, which we address in Sect. 3, occur when the supporting surface has non-empty boundary components and the resulting edge-biregular maps can then have non-even valency or face length. For example, if in an edge-biregular map a vertex and its incident bold edge, and hence all bold edges, lie on the boundary of the surface, then the vertices will necessarily have odd degree, 3. See Bryant and Singerman [3] for "Foundations of the theory of maps on surfaces with boundary".

Each edge-biregular map $M=\left(H ; r_{0}, r_{2}, \rho_{0}, \rho_{2}\right)$ has a twin map which is the same as the original map in every respect except the colouring of the edge orbits is switched. Since each generator is associated with one of the colours of the edges, the twin map of $M$ is denoted $W=\left(H ; \rho_{0}, \rho_{2}, r_{0}, r_{2}\right)$. This is just a matter of relabelling and does not imply or demand any further relationship between the two structures. 


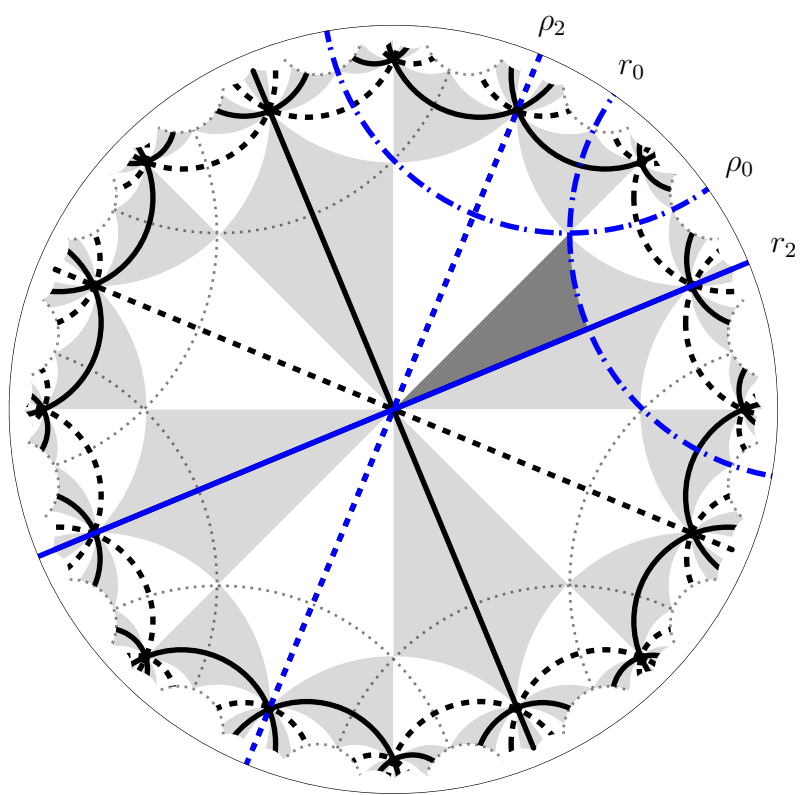

Fig. 4 The hyperbolic lines of reflection for the generating automorphisms $r_{0}, r_{2}, \rho_{0}$ and $\rho_{2}$, shown on part of the infinite edge-biregular map (hyperbolic tessellation) of type $(8,4)$. The marked corner, corresponding to the identity element of $H$, is shaded darker than the others

The map automorphisms in $H$ of an edge-biregular map are those which are generated by the four reflections along and across each of two particular adjacent edges of the map, as shown in Fig. 4. As we have seen the group $H$ acts regularly on the set of corners of the map. It is possible that the map has further symmetries, and the established definition for the full automorphism group of a map is defined with respect to its action on the set of flags of the map, with no reference to the colouring of edges, see [9]. When considered as acting in the natural way on the flags of the map, the group $H=\left\langle r_{0}, r_{2}, \rho_{0}, \rho_{2}\right\rangle$ partitions the flag set of a given edge-biregular map into two orbits, one containing shaded flags, and the other containing unshaded flags. As such, it is possibly a 2-orbit map, one of those classified by Hubard, Orbanić and Weiss [8]. However, an edge-biregular map may not be a 2-orbit map, since there is no structure within our definition which disallows a colour-reversing automorphism of the map. Such an automorphism, if it exists, would fuse the two edge orbits together and the full automorphism group for the map would then be transitive on flags, making it a fully regular map, and in this case the full automorphism group would then contain $H$ as an index two subgroup. The edge-biregular map $M=\left(H ; r_{0}, r_{2}, \rho_{0}, \rho_{2}\right)$ is thus a fully regular map if and only if there is an involutory automorphism $\psi$ of the group $H$ such that $\psi: r_{i} \leftrightarrow \rho_{i}$ for each $i \in\{0,2\}$.

Two edge-biregular maps $M=\left(H ; r_{0}, r_{2}, \rho_{0}, \rho_{2}\right)$ and $M^{\prime}=\left(H^{\prime} ; r_{0}^{\prime}, r_{2}^{\prime}, \rho_{0}^{\prime}, \rho_{2}^{\prime}\right)$ are isomorphic to each other if and only if the mapping $r_{i} \rightarrow r_{i}^{\prime}$ and $\rho_{i} \rightarrow \rho_{i}^{\prime}$ for $i \in\{0,2\}$ extends to an isomorphism of the group $H$. Thus, an edge-biregular map $M$ is a fully regular map if and only if it is isomorphic to its twin. 


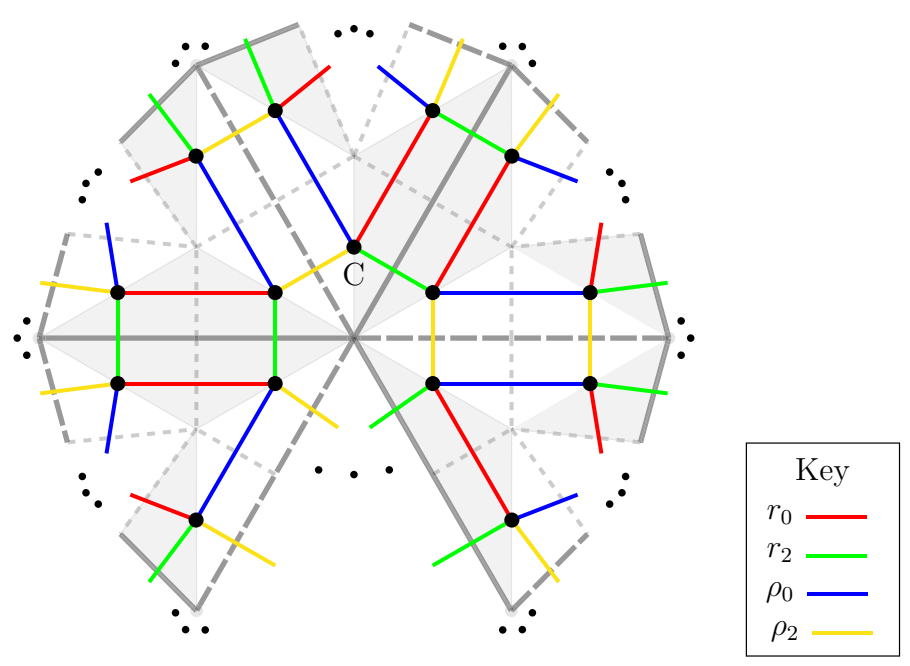

Fig. 5 Part of the Cayley map

Any edge-biregular map which is not fully regular is an example of a $k$-orbit map for $k=2$, see [12]. Edge-biregular maps which are not fully regular are the two-orbit maps of type 20,2 classified in [8]. In [9], Jones also makes special mention of this class of maps as it is the only non-edge-transitive class which arises very naturally in the process of determining the 14 classes of edge-transtitive maps. These types of edge-transitive maps were classified in different terms by Graver and Watkins in [7] and Wilson in [15].

The choice of notation indicates some connection between the corner-monodromy group $G$ and $H$, and indeed there is a very natural relationship between the two. The groups $H$ and $G$ are regular permutation representations on the set of corners of the coloured map acting, respectively, on the left and right. The embedded Cayley map illustrates this in Fig. 5. This is a Cayley graph for the automorphism group $H$, so each of the dark vertices represents an element in $H$, naturally identified with the corners of the edge-biregular map, and the coloured lines are the generating automorphisms $r_{0}, r_{2}, \rho_{0}$ and $\rho_{2}$. Take the vertex in the corner marked $C$ as the identity element of the group $H$ and consider the element (for example) $h=\rho_{2} r_{2} r_{0} \rho_{0} \in H$. Now, $H$ acts on the left, so $h$ corresponds to the flag which is the image of $C$ after the reflections $\rho_{0}, r_{0}, r_{2}$ and $\rho_{2}$ are applied in that order. Applying these automorphisms in turn can be a somewhat laborious exercise.

However, one arrives at the same corner as when going from $C$ to $C \mathcal{P}_{2} \mathcal{R}_{2} \mathcal{R}_{0} \mathcal{P}_{0}$. This is no coincidence. Notice that the coloured lines, when looked at in the context of the underlying map shown in grey, indicate the gluing instructions $\mathcal{R}_{0}, \mathcal{R}_{2}, \mathcal{P}_{0}, \mathcal{P}_{2}$ between the corners. An automorphism $h x \in H$ for some $x \in\left\{\rho_{2}, r_{2}, r_{0}, \rho_{0}\right\}$ acts on the marked corner $C$ as follows. Being an automorphism, $x$ commutes with the monodromy group, and $C$ is our marked corner, so $x(C) \mathcal{X}=x(C \mathcal{X})=C$ where $\mathcal{X}$ is the corresponding capital of $x$. This implies that $x(C)=C \mathcal{X}$ and hence, $h x(C)=$ $h(C) \mathcal{X}$. By induction, we can conclude that, where the corner $C$ is identified with 
the identity of $H$, the automorphism $h=x_{1} x_{2} \ldots x_{n} \in H$ is identified with the flag $C \mathcal{X}_{1} \mathcal{X}_{2} \ldots \mathcal{X}_{n}$

\subsection{Algebraic context}

Maps of a given type $(k, l)$ can be obtained as quotients of the universal map of the same type. This universal map consists of the regular tessellation of $l$-gons, $k$ of which meet at each corner, on a simply connected surface. The corresponding $(k, l)$ tessellation is described as hyperbolic, Euclidean or spherical, the name describing the geometry of the underlying simply connected surface.

The universal map of type $(k, l)$ is known [13] to have automorphism group as follows:

$$
T_{k, l}=\left\langle R_{0}, R_{2}, R_{1} \mid R_{0}^{2}, R_{2}^{2}, R_{1}^{2},\left(R_{0} R_{2}\right)^{2},\left(R_{1} R_{2}\right)^{k},\left(R_{0} R_{1}\right)^{l}\right\rangle
$$

The group $T_{k, l}$ is called the full triangle group of type $(k, l)$, and it is finite only for maps of the spherical type, where $1 / k+1 / l>1 / 2$. Quotients of the full triangle group by torsion-free normal subgroups of finite index give rise to finite fully regular maps, that is maps where the automorphism group acts transitively on the finite set of flags.

When $k$ and $l$ are both even, there are seven index two subgroups of $T_{k, l}$, each of which is a source of maps with a given property. These index two subgroups can be identified by listing which of the three generators are included, and we use bar notation to indicate when the corresponding element is not contained in the subgroup. Well-known, and widely studied are the rotary maps which occur as quotients of the index two subgroup in which none of the generators are included, that is $T_{k, l}^{+}=$ $\left\langle R_{0} R_{1}, R_{2} R_{1}\right\rangle=\left\langle\bar{R}_{0}, \bar{R}_{1}, \bar{R}_{2}\right\rangle$. Recently, Breda, Catalano and Širán̆ [5] have partially classified the bi-rotary maps which come from quotients of $\left\langle R_{0}, \bar{R}_{2}, \bar{R}_{1}\right\rangle$. The maps which are the focus of this paper, that is edge-biregular maps of type $(k, l)$, arise as quotients of the index two subgroup $\left\langle R_{0}, R_{2}, \bar{R}_{1}\right\rangle$.

We note that edge-biregular maps can occur as a special case of the 2-regular hypermaps in the "edge-bipartite" class as described in Duarte's thesis [6], where there is a classification for some surfaces of small Euler characteristic. In this paper, we allow for maps with semi-edges, making our work more general in this sense.

\section{Special cases of edge-biregular maps}

An edge-biregular map is usually described in terms of the four generating automorphisms $r_{0}, r_{2}, \rho_{0}, \rho_{2}$, but there are degenerate cases where either one or more of these is "missing" (contributing nothing to the group, and indicating a boundary to the surface) or when the four involutions are not distinct. These possibilities are explored in this section. 


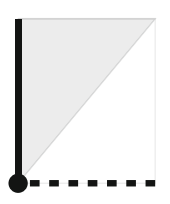

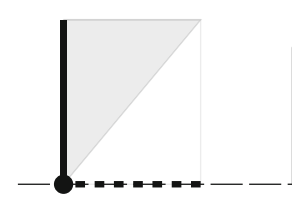

(a)

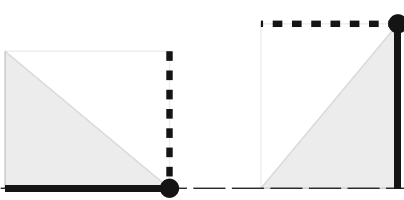

(c)

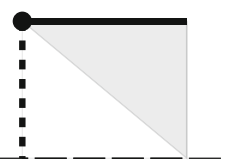

(d)

Fig. 6 A corner, and corners meeting the surface boundary, types (a), (b), (c), and (d)
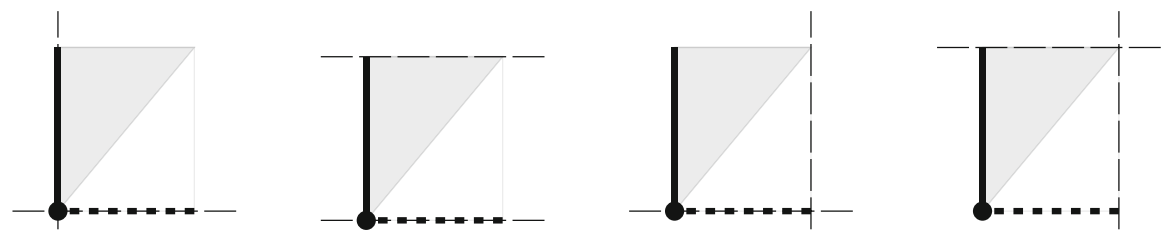

Fig. 7 Corner regions meeting the surface boundary in two ways (up to colouring)

\subsection{Edge-biregular maps with semi-edges}

A way in which the four involutions might not be distinct would be if $r_{0}=r_{2}$ and/or $\rho_{0}=\rho_{2}$. This would indicate that the orbit of edges of the corresponding colour consists of semi-edges.

If both orbits of edges are semi-edges, then we have a semistar. These maps have only one vertex, and, assuming there are more than two corners in the map, the group $H=\left\langle r_{2}, \rho_{2}\right\rangle$ will be dihedral. The supporting surface, if without boundary, must be a sphere, and these even valency semistar spherical maps are both edge-biregular and also fully regular. Examples of semistar edge-biregular maps also exist on a disc, and we explore surfaces with boundary components in the following section.

Henceforth, we will assume, up to twinness, that at least one of the orbits of edges does not consist of semi-edges. When there are no semi-edges in an edge-biregular map, we say it is a proper edge-biregular map.

\subsection{Edge-biregular maps with boundary components}

If a map is on a surface which is not closed, that is when the surface has non-empty boundary components, then at least one of the edges of a corner region will lie on the boundary of the supporting surface. The resulting maps are reminiscent of maps with holes, see page 109 in [4], and holey maps, see [2]. Figure 6 shows a corner region, and ways that corner regions can meet the boundary of a surface. Any given corner region is the union of two adjacent flags and so can be represented by a quadrangle in the natural way. Hence, a corner region could have up to four of its edges along the boundary, see Figs. 7 and 8. For clarity of the diagrams, we have drawn the boundary lines extending beyond the corner regions. In actuality, due to the map being on a 2-manifold with boundary (where every point on a boundary has a neighbourhood homeomorphic to a half-disc), wherever two boundaries apparently cross, the boundary will in fact be one continuous boundary around this part of the corner region. 

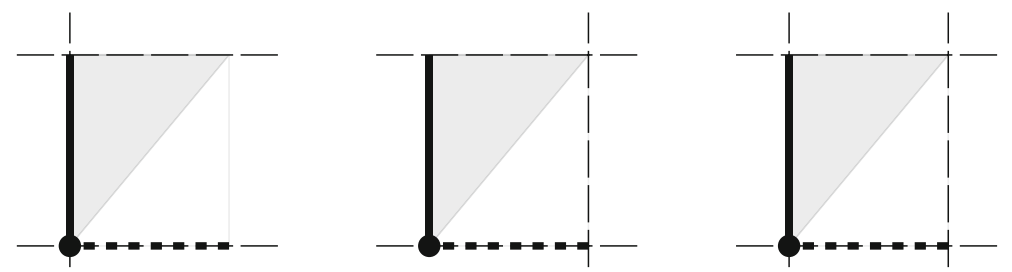

Fig. 8 Corners meeting the surface boundary in three (up to colouring) or four ways
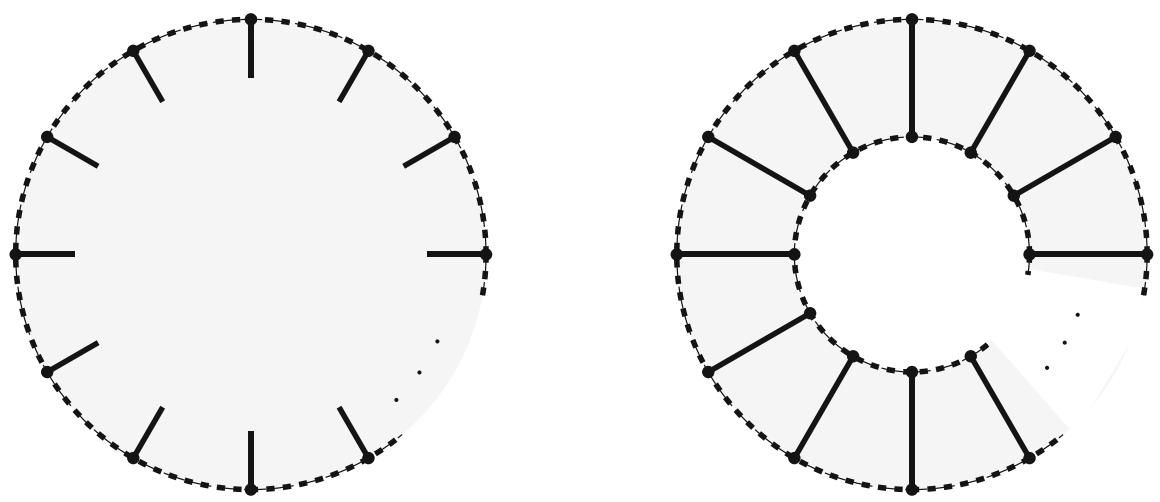

Fig. 9 Edge-biregular maps with unshaded edges along the surface boundary

Edge-biregular maps with boundary occur when one or more of the generators (and hence also the relators including them) collapse and hence are missing from the usual group presentation $H=\left\langle r_{0}, r_{2}, \rho_{0}, \rho_{2}\right| r_{0}^{2}, r_{2}^{2}, \rho_{0}^{2}, \rho_{2}^{2}\left(r_{0} r_{2}\right)^{2},\left(\rho_{0} \rho_{2}\right)^{2}$, $\left.\left(r_{2} \rho_{2}\right)^{k / 2},\left(r_{0} \rho_{0}\right)^{l / 2}, \ldots\right\rangle$. For some $x \in\{r, \rho\}$ if $x_{0}$ is missing, then the corresponding coloured edge (and so all such edges) will in fact be a semi-edge to the boundary, while if $x_{2}$ is missing, then all the edges with the corresponding colour will be along the surface boundary. If, for example, the unshaded edges are on the surface boundary, then $\rho_{2}$ is missing and we would denote the edge-biregular map by $M=\left(H ; r_{0}, r_{2}, \rho_{0}, \overline{\rho_{2}}\right)$.

Considering an edge-biregular map $M=\left(H ; r_{0}, r_{2}, \rho_{0}, \overline{\rho_{2}}\right)$, like type (a) in Fig. 6 , we have, by regularity of the action of $H$ on corners of the map, all the unshaded edges must be on the boundary of the map. Let us assume, for the moment, that this is the only way in which corner regions meet the surface boundary. When the bold edges are semi-edges, which means that $r_{0}=r_{2}$, the group $H=\left\langle r_{0}, \rho_{0}\right\rangle$ is dihedral, and the edge-biregular map has a single face with the supporting surface being a disc. This is illustrated in Fig. 9, on the left. When the bold edges are not semi-edges, then $l=4$ and the underlying graph is a ladder so the surface is homeomorphic to either an annulus or a Möbius strip. These maps can have any number of bold (semi)edges, and generalisations are shown in Fig. 9, where the orientability of the second surface is dependent on the identification of edges (and the relators in the group presentation) where the dots are. 
Notice that the vertices of these edge-biregular maps have odd valency. This is no surprise since having the vertices on the surface boundary destroys any hope of a non-trivial rotation around a vertex being an automorphism of the map.

Thinking about these edge-biregular maps in terms of their group presentations yields something even more interesting. Type (a) has the following partial presentation:

$$
H=\left\langle r_{0}, r_{2}, \rho_{0} \mid r_{0}^{2}, r_{2}^{2}, \rho_{0}^{2},\left(r_{0} r_{2}\right)^{2},\left(r_{0} \rho_{0}\right)^{l / 2}, \ldots\right\rangle
$$

and we are left with $H$ being a group which is generated by three involutions, two of which commute. This phrase, in italics, will be very familiar to those who study fully regular maps, as this is precisely how the automorphism group of a fully regular map is often described. The group $H$ is, in this case, the automorphism group of a fully regular map. While the above presentation of the group $H$ describes an edge-biregular map of type (a), it also resembles the usual partial presentation for the automorphism group of a fully regular map, with $\rho_{0}$ in place of $r_{1}$. This indicates a very close relationship between the two corresponding maps which is as follows.

Construction 1 Starting with a fully regular map $\left(G ; r_{0}, r_{2}, r_{1}\right)$ of type $(k, l)$, see [13] for details, choose an arbitrary vertex $v$ and cut out a small disc neighbourhood around $v$ (the disc must be small enough not to include any other vertices nor edges that are not incident to $v$ ). Place new vertices where edges meet this new surface boundary and draw unshaded edges between the new vertices all along this new surface boundary. Repeating this process for all vertices of the original fully regular map will yield the well-defined edge-biregular map $\left(H ; r_{0}, r_{2}, \rho_{0}, \overline{\rho_{2}}\right)$ of type $(3,2 l)$ with $\rho_{0}=r_{1}$. In the other direction, starting with an edge-biregular map with the unshaded edges along a surface boundary, the related regular map can be built by contracting each unshaded edge down into a single vertex. By letting all the unshaded edges (and hence, flags, and indeed boundaries) disappear in the process, the reflections which used to act along the unshaded edges (conjugates of $\rho_{0}$ ) now simply act as the reflections across the resulting new corners (that is, conjugates of $r_{1}$ ) of the implicit fully regular map.

An edge-biregular map with boundary of type (b) is the twin of a map of type (a), while edge-biregular maps with boundary types (c) and (d) also form twin pairs. Figure 10 shows some edge-biregular maps of type (c), the oppositely coloured twins of type (d) edge-biregular maps.

If one of the edges of a particular colour, let us say unshaded, meets the boundary, like (d) in Fig. 6, then this edge, and hence, all edges of this type are semi-edges to a boundary of the surface. This is an edge-biregular map $\left(H ; r_{0}, r_{2}, \overline{\rho_{0}}, \rho_{2}\right.$, $)$. Assuming that a corner region meets the boundary only this way, and the shaded edges are not semi-edges (otherwise, we would end up with a semistar), then each face region of the map must have as its boundary: one shaded edge; two unshaded semi-edges; and a section of the surface boundary. This type (d) edge-biregular map thus has presentation:

$$
H=\left\langle r_{0}, r_{2}, \rho_{2} \mid r_{0}^{2}, r_{2}^{2}, \rho_{2}^{2},\left(r_{0} r_{2}\right)^{2},\left(r_{2} \rho_{2}\right)^{k / 2}, \ldots\right\rangle
$$



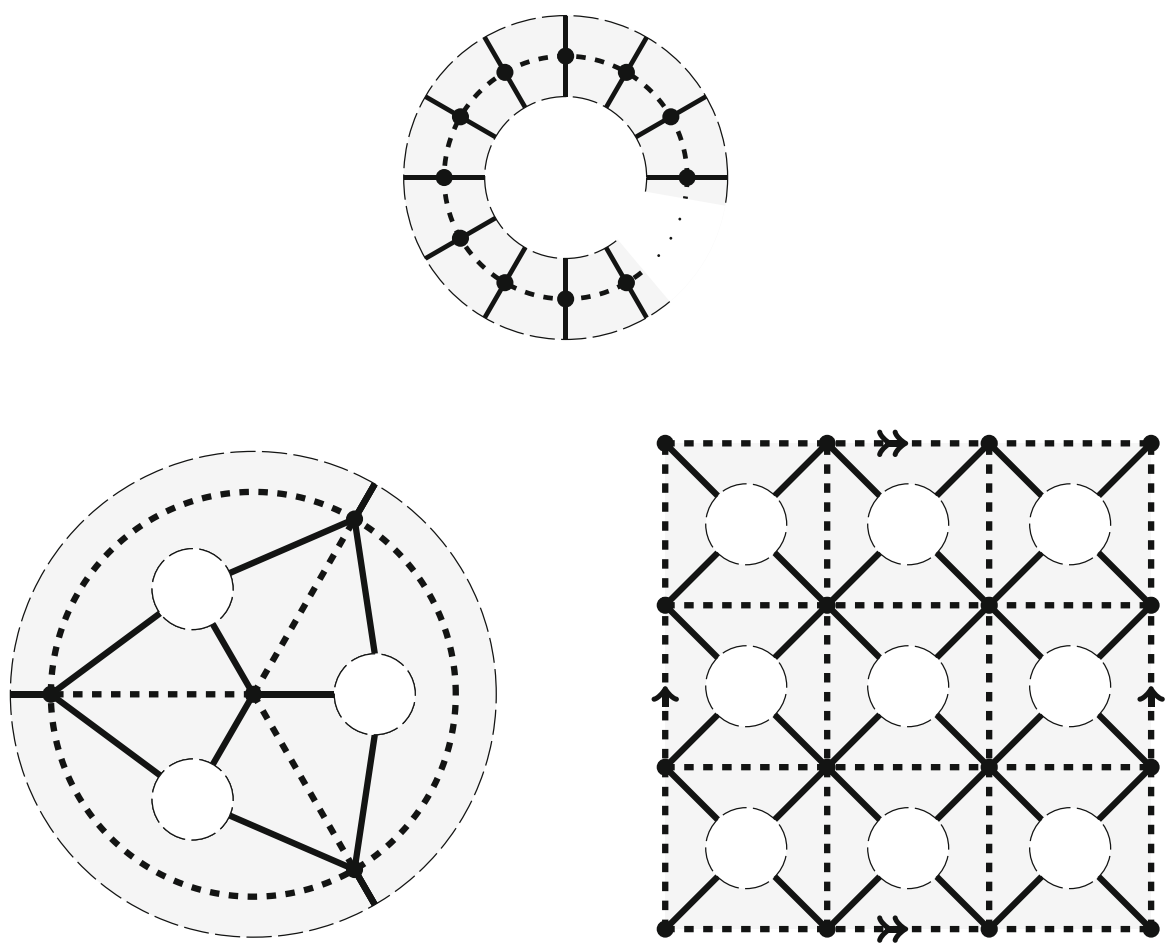

Fig. 10 Edge-biregular maps of type (c) with semi-edges meeting the surface boundaries

and we are again left with $H$ being a group which is generated by three involutions, two of which commute. This time we have $\rho_{2}$ instead of $r_{1}$, and this yields the following construction.

Construction 2 Starting from a regular map, $\left(G ; r_{0}, r_{2}, r_{1}\right)$, drawn with all its edges shaded, we can obtain the well-defined implied edge-biregular map $\left(H ; r_{0}, r_{2}, \overline{\rho_{0}}, \rho_{2},\right)$ by cutting a disc out from the interior of each face and drawing an unshaded semi-edge from this new surface boundary to each of the surrounding vertices, thereby letting $r_{1}=\rho_{2}$. Conversely, given an edge-biregular map with unshaded semi-edges to the surface boundary, we can obtain a regular map by deleting all the unshaded semiedges and contracting each of the boundaries in the surface to ensure the interior of each resulting face is homeomorphic to an open disc. This valid since the boundary component which a semi-edge meets must be the same as the boundary component which the semi-edge from the adjacent corner within the same face meets.

If a corner meets the boundary in more than one of the ways listed (as in the diagrams in Figs. 7 and 8), then options are severely restricted: there are at most two involutory generators and so the group $H$ is dihedral or cyclic. The interested reader may wish to verify that these include the single faced maps on a disc $H=\left\langle r_{0}, \rho_{0}\right\rangle$, the edge-biregular maps on an orientable band $H=\left\langle r_{2}, \rho_{0}\right\rangle$, the 4-cornered map on a 
$\operatorname{disc} H=\left\langle r_{0}, r_{2}\right\rangle \cong V_{4}$ and the semistar on a disc $H=\left\langle r_{2}, \rho_{2}\right\rangle$ as well as their twins, and the more trivial maps on a disc which have only one or two corners.

\subsection{Edge-biregular maps with non-distinct generators}

There is another natural, if somewhat trivial way of building an edge-biregular map from any given fully regular map, this time without introducing any boundaries, and that is as follows.

Construction 3 Given a fully regular map $\left(G ; r_{0}, r_{2}, r_{1}\right)$ of type $(k, l)$ drawn with all its edges shaded. At every vertex draw $k$ unshaded semi-edges, so that there is one semi-edge in every corner of the original map. This is thus the edge-biregular map $\left(H ; r_{0}, r_{2}, \rho, \rho\right)$ where $\rho=\rho_{0}=\rho_{2}=r_{1}$.

Figure 11 shows a cube (a fully regular map) drawn with all shaded edges, along with the edge-biregular maps as described in the three constructions above. If you draw the embedded Cayley graphs for each of these maps, the close relationships between this collection of four maps becomes very clear. We could of course include the corresponding twin maps too, as well as the dual maps, and hence, each fully regular map has many edge-biregular related maps.

Having addressed maps with non-empty boundary components in the previous section, henceforth we will assume that the supporting surfaces for our edge-biregular maps are closed, that is without boundary.

There are other degeneracies arising from non-distinct generators, and we will now address these in turn. Up to duality and twinness, we may assume that one of the non-distinct generators is $r_{0}$, and so as to avoid bold semi-edges, we assume the other is in $\left\{\rho_{0}, \rho_{2}\right\}$.

In the case when $r_{0}=\rho_{0}$, we have $H=\left\langle r_{0}, r_{2}, \rho_{2}\right\rangle$, the group for an edge-biregular map which has digonal faces. Maps of type $(k, 2)$ are known to be fully regular and are supported by either the sphere or the projective plane.

Construction 4 The edge-biregular map $\left(H ; \rho, r_{2}, \rho, \rho_{2}\right)$ where $\rho=r_{0}=\rho_{0}$ has an implied regular map $\left(G ; r_{0}, r_{2}, r_{1}\right)$ where $r_{1}=\rho_{2}$ which is an embedding of the bold edges of the edge-biregular map, with the dashed edges deleted. This implied fully regular map also has digonal faces. In the other direction, a fully regular map with digonal faces can be made into an edge-biregular map by the addition of unshaded edges, each cutting each original digonal face into two alternate-edge-coloured digons.

Remark 3.1 It may be tempting to think that any edge-biregular map with non-distinct generators corresponds to a fully regular map with the same automorphism group and supported by the same surface. However, this is not the case as will now become clear.

In the case where $r_{0}=\rho_{2}=\rho$, we have the edge-biregular map $\left(H ; \rho, r_{2}, \rho_{0}, \rho\right)$ while the group $H=\left\langle r_{0}, r_{2}, \rho_{0}\right\rangle$. Geometrically, $r_{0}=\rho_{2}$ implies that (all) the edges in the bold orbit are loops. Also, $r_{0} r_{2}=\rho_{2} r_{2}$ has order dividing two and hence the vertices all have degree ( 2 or) 4 . Similarly, $\rho_{0} \rho_{2}=\rho_{0} r_{0}$ has order dividing two, and so (assuming no further degeneracies occur) we have an edge-biregular map of 

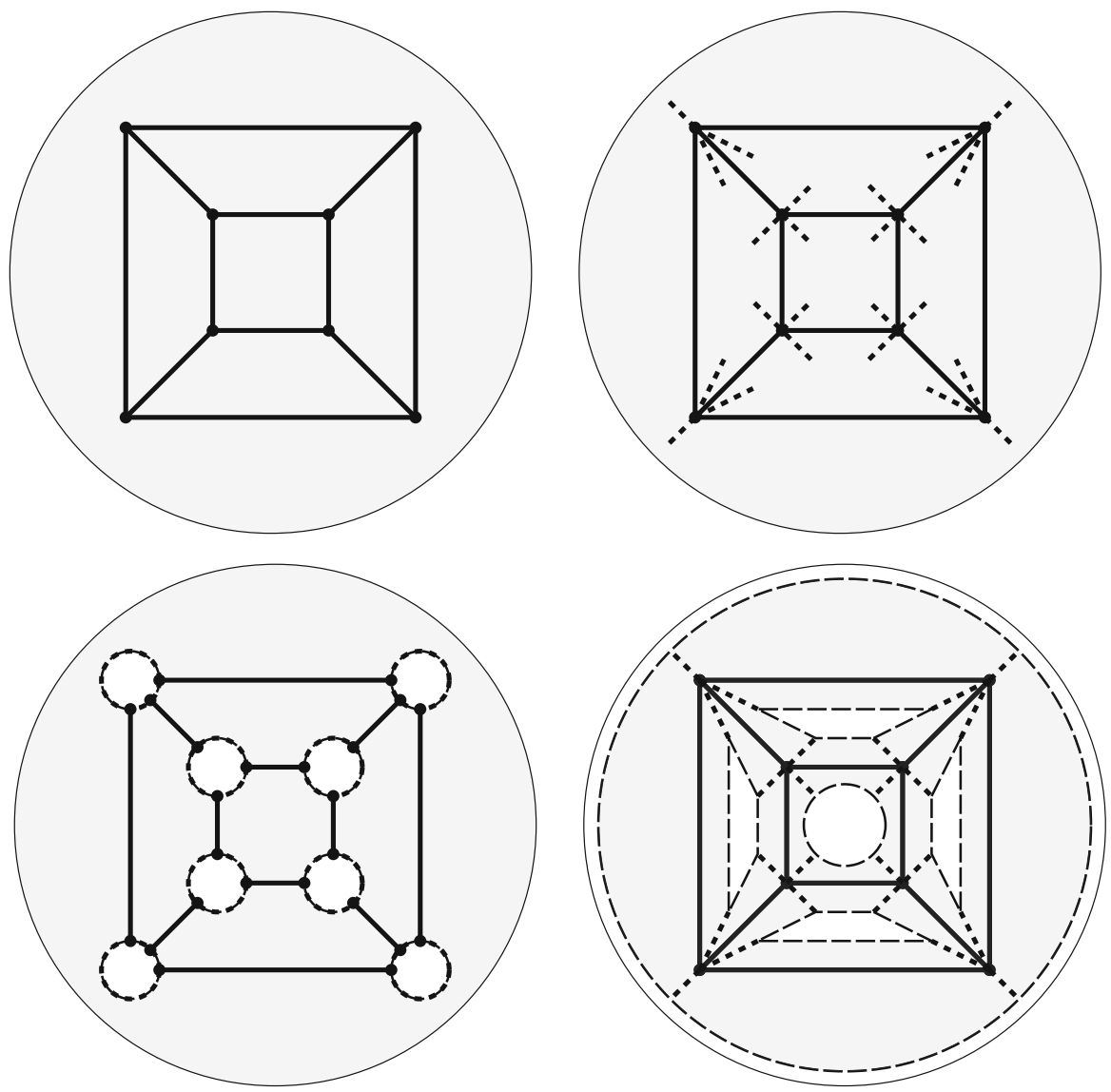

Fig. 11 A fully regular map and some of its associated edge-biregular maps

type $(4,4)$. Now, the tessellation of type $(4,4)$ is Euclidean, indicating that the maps $\left(H ; \rho, r_{2}, \rho_{0}, \rho\right)$ supported by a closed surface must be, depending on orientability, on either the Klein bottle or the torus. Note: We present a classification of proper edge-biregular maps on the torus and the Klein bottle in Sects. 4.2.1 and 4.2.2.

Treating $r_{0}$ and $r_{2}$ in the usual way, we consider the implications of $\rho_{0}$ being thought of as $r_{1}$, the reflection in a corner of a regular map. Informally, this is like reducing (the marked one, and hence all) the dashed edges to negligible length, thereby identifying the endpoints of each dashed edge and stitching the remaining shaded flags together in the correspondingly natural way. The resulting fully regular map would therefore have faces which are digons and the underlying graph would consist of just one vertex. Thus, the underlying graph has become a bouquet of loops, but this process has also drastically changed the underlying surface, as we might have expected if we remembered that the Klein bottle has no regular map. In the case of the Klein bottle, the resulting surface is the projective plane. However, in the case of a toroidal map, the object created by this process (which is equivalent to contracting a non-contractible 
cycle) is in fact a pseudo-surface, a sphere with one pair of antipodal points identified, in which case the resulting map is an example of a regular pinched map, see [1] for further details.

\section{Edge-biregular maps on closed surfaces where $\chi \geq 0$}

\subsection{Euler's formula}

Consider the edge-biregular map $\left(H ; r_{0}, r_{2}, \rho_{0}, \rho_{2}\right)$ which has type $(k, l)$, and no semi-edges.

The stabiliser for the distinguished face is denoted $D_{l}:=\left\langle r_{0}, \rho_{0}\right\rangle$ and is isomorphic to the dihedral group with $l$ elements, while the stabiliser for the distinguished vertex is called $D_{k}:=\left\langle r_{2}, \rho_{2}\right\rangle$ and is isomorphic to the dihedral group with $k$ elements. The map thus has $\frac{|H|}{l}$ faces and $\frac{|H|}{k}$ vertices.

The stabiliser group of an edge is isomorphic to $V_{4}$ and, for the distinguished shaded edge is $\left\langle r_{0}, r_{2}\right\rangle$, while for the distinguished unshaded edge the stabiliser is $\left\langle\rho_{0}, \rho_{2}\right\rangle$. Hence, the map has $\frac{2|H|}{4}$ edges.

Supposing that the map $\left(H ; r_{0}, r_{2}, \rho_{0}, \rho_{2}\right)$ lies on a surface of Euler characteristic $\chi$, we apply the well-known Euler-Poincaré formula, which is useful when we come to classifying these maps on particular surfaces:

$$
\chi=|H|\left(\frac{1}{k}-\frac{1}{2}+\frac{1}{l}\right)
$$

When applying Euler's formula to a map with semi-edges, one should realise that the existence of semi-edges contribute nothing to the value of $\chi$.

\subsection{Edge-biregular maps of non-negative Euler characteristic}

In this section, we classify all edge-biregular maps on surfaces for which $\chi \in\{0,1,2\}$. We note that Duarte's thesis [6] includes a classification of 2-restrictedly-regular edgebipartite hypermaps, that includes all proper edge-biregular maps, for the sphere, the projective plane and the torus. Here, we allow for the possibility of semi-edges, and we present a different approach for Euclidean edge-biregular maps. While Duarte's work allows for many different group presentations which describe the same Euclidean map, our approach standardises the presentation for such an edge-biregular map and extends to include a classification for such maps on the Klein bottle.

Theorem 4.1 If the proper edge-biregular map $M=\left(H ; r_{0}, r_{2}, \rho_{0}, \rho_{2}\right)$ has nondistinct generators, then it is supported by a surface which has non-negative Euler characteristic. Up to duality, the surfaces, maps and their groups $H$ are listed below:

1. Sphere: dipoles of even degree with $H=\left\langle r_{2}, \rho_{2}\right\rangle \times\left\langle\rho_{0}\right\rangle=D_{k} \times\left\langle\rho_{0}\right\rangle$ and $r_{0}=\rho_{0}$.

2. Projective plane: single vertex maps, the degree $k$ being a multiple of 4 , with $H=\left\langle r_{2}, \rho_{2}\right\rangle=D_{k}$ and $r_{0}=\rho_{0}$. 
3. Torus: Maps of type $(4,4)$ with $m$ quadrangular faces and (at least) one edge orbit consisting of loops such that $H=\left\langle r_{2}, \rho_{0}\right\rangle \times\left\langle r_{0}\right\rangle \cong D_{2 m} \times C_{2}$ and $r_{0}=\rho_{2}$.

4. Klein bottle: Maps of type $(4,4)$ with $m / 2$ quadrangular faces and (at least) one edge orbit consisting of loops such that $H=\left\langle r_{2}, \rho_{0}\right\rangle \cong D_{2 m}$ and $r_{0}=\rho_{2}$.

Proof Suppose we have a proper edge-biregular map $M=\left(H ; r_{0}, r_{2}, \rho_{0}, \rho_{2}\right)$ such that the generators $r_{0}, r_{2}, \rho_{0}, \rho_{2}$ are not distinct. The map is assumed to be proper, so there are no semi-edges which forces $r_{0} \neq r_{2}$ and $\rho_{0} \neq \rho_{2}$. Up to duality, we may assume that one of the redundant generators is labelled $r_{0}$. This leaves two options, $r_{0}=\rho_{0}$, or $r_{0}=\rho_{2}$, which we will address in turn.

If $r_{0}=\rho_{0}$, then the element $r_{0} \rho_{0}$ is the identity which means $l=2$ and the faces of the map are digons. Also note that $H=\left\langle r_{0}, r_{2}, \rho_{0}, \rho_{2}\right\rangle=\left\langle r_{2}, \rho_{0}, \rho_{2}\right\rangle$ and we have $\left[\rho_{0}, r_{2}\right]$ as well as $\left[\rho_{0}, \rho_{2}\right]$ so $\rho_{0}$ is central. Hence, either $H=D_{k}$ or $H=D_{k} \times$ $\left\langle\rho_{0}\right\rangle$. Applying the Euler-Poincare formula, we see that these two cases correspond, respectively, to a single-vertex degree- $k$ map on the projective plane (remembering $4 \mid k$ ), and a degree- $k$ dipole embedded in the sphere (for any even $k$ ).

If $r_{0}=\rho_{2}$, then the bold edges must be loops. Also, we have $\left[\rho_{0}, \rho_{2}\right]=\left[\rho_{0}, r_{0}\right]$ so $l=4$ and $\left[r_{0}, r_{2}\right]=\left[\rho_{2}, r_{2}\right]$ so $k=4$ and hence, the map type is $(4,4)$. This implies we are on a surface with Euler characteristic $\chi=0$. If the surface is orientable, then the map is on the torus, otherwise the map is supported by the Klein bottle. Going further, in the case where $r_{0}=\rho_{2}$, notice that $r_{0}$ is central, $H=\left\langle r_{0}, r_{2}, \rho_{0}\right\rangle$, and also that $\left\langle r_{2}, \rho_{0}\right\rangle$ is a dihedral group, with order, say, $2 m$. Now, if $r_{0} \in\left\langle r_{2}, \rho_{0}\right\rangle$, then $H=\left\langle r_{2}, \rho_{0}\right\rangle \cong D_{2 m}$ where $m$ is even. Also, being central, $r_{0}=\left(r_{2} \rho_{0}\right)^{m / 2}$ which yields a relator of odd length, forcing the supporting surface to be non-orientable, the Klein bottle. If, on the other hand, $r_{0} \notin\left\langle r_{2}, \rho_{0}\right\rangle$ then $H=\left\langle r_{2}, \rho_{0}\right\rangle \times\left\langle r_{0}\right\rangle \cong D_{2 m} \times C_{2}$ and, as the direct product of a dihedral group (with the presentation generated by two involutions) and a copy of $C_{2}$, there can be no relators of odd length. These maps are therefore supported by an orientable surface of Euler characteristic 0, namely the torus.

The workings in the above proof have shown that when $H$ has non-distinct generators $r_{0}, r_{2}, \rho_{0}, \rho_{2}$, then the closed supporting surface for the edge-biregular map has non-negative Euler characteristic $\chi \in\{0,1,2\}$. This yields the following corollary on which we will rely later.

Corollary 4.2 A proper edge-biregular map $M=\left(H ; r_{0}, r_{2}, \rho_{0}, \rho_{2}\right)$, embedded on a surface where $\chi<0$, has distinct generators $r_{0}, r_{2}, \rho_{0}, \rho_{2}$.

\subsubsection{Edge-biregular maps on the torus}

Each regular map on the torus will have associated edge-biregular maps each created by inserting a semi-edge into each corner. Thus, there will be edge-biregular maps of types $(12,6),(6,12)$ and $(8,8)$ derived by construction 3 , respectively, from the well-known toroidal regular maps of types $(6,3),(3,6)$ and $(4,4)$.

Turning our attention to proper edge-biregular maps supported by the torus, the requirement for even valency and face length makes it clear that they must all have 
type $(4,4)$. The infinite group

$$
\Delta=\left\langle R_{0}, R_{2}, P_{0}, P_{2} \mid R_{0}^{2}, R_{2}^{2}, P_{0}^{2}, P_{2}^{2},\left(R_{0} R_{2}\right)^{2},\left(P_{0} P_{2}\right)^{2},\left(R_{0} P_{0}\right)^{2},\left(R_{2} P_{2}\right)^{2}\right\rangle
$$

describes the colour-preserving automorphism group of the alternate-edge-coloured infinite square grid, a tessellation of type $(4,4)$ on the Euclidean plane. Following the work in the seminal paper by Jones and Singerman [10], the colour-preserving automorphism group $H$ for any given finite proper edge-biregular map of type $(4,4)$ arises as a smooth quotient of $\Delta$ by a torsion-free normal subgroup $N$ of finite index giving $H \cong \Delta / N$.

Using an analogue of the example found in Section 7 of [10], to assist in identifying subgroups of finite index in $\Delta$, we now define $T:=\left\langle R_{0} P_{2}, R_{2} P_{0}\right\rangle \leq \Delta$, noting that the two generators, each of which has infinite order, commute and so $T$ is itself a free abelian group of rank two, $T \cong Z \times Z$. Conjugation by any one of the elements $R_{0}, R_{2}, P_{0}$, or $P_{2}$ fixes the subgroup $T$ so it is easy to see that $T$ is normal in $\Delta$. For example, $R_{0}$ inverts $R_{0} P_{2}$ and fixes $R_{2} P_{0}$, while conjugation by $R_{2}$ fixes $R_{0} P_{2}$ and inverts $R_{2} P_{0}$, and this yields $\Delta=T \rtimes\left\langle R_{0}, R_{2}\right\rangle \cong(Z \times Z) \rtimes V_{4}$.

This presentation of $\Delta$ makes it clear that any rank two (torsion-free) subgroup of $T$ will be of finite index in $\Delta$. Geometrically speaking, the group $T$ describes all the translations which map the aforementioned infinite square grid to itself. It will be helpful later to have the following visualisation in mind: if we mark an arbitrary vertex of the infinite grid, any subgroup of $T$ generated by a given pair of independent translations creates a two-dimensional lattice of equivalent vertices, all of which correspond to a single vertex on the associated toroidal map. It is well-known that two linearly independent translations of the plane determine a fundamental region for a torus. Drawing this all together, we have that $N_{T}$, a given rank two subgroup of $T$, gives rise to an edge-biregular map on a torus if and only if $N_{T} \unlhd \Delta$.

Although there may be many equivalent presentations of the group $H$, each of which describes a particular given edge-biregular map on a torus, the following proposition seeks to identify a uniquely defined presentation corresponding to each toroidal edgebiregular map.

Proposition 4.3 Every proper toroidal edge-biregular map is determined by the group $H_{. .}$with one of the two following presentations:

$$
H_{\text {Rect }}=\left\langle r_{0}, r_{2}, \rho_{0}, \rho_{2} \mid r_{0}^{2}, r_{2}^{2}, \rho_{0}^{2}, \rho_{2}^{2},\left(r_{0} r_{2}\right)^{2},\left(\rho_{0} \rho_{2}\right)^{2},\left(r_{0} \rho_{0}\right)^{2},\left(r_{2} \rho_{2}\right)^{2},\left(r_{0} \rho_{2}\right)^{a},\left(r_{2} \rho_{0}\right)^{c}\right\rangle
$$

or

$$
\begin{aligned}
H_{\text {Rhomb }}= & \left\langle r_{0}, r_{2}, \rho_{0}, \rho_{2}\right| r_{0}^{2}, r_{2}^{2}, \rho_{0}^{2}, \rho_{2}^{2},\left(r_{0} r_{2}\right)^{2},\left(\rho_{0} \rho_{2}\right)^{2},\left(r_{0} \rho_{0}\right)^{2},\left(r_{2} \rho_{2}\right)^{2}, \\
& \left.\left(r_{0} \rho_{2}\right)^{2 b},\left(r_{0} \rho_{2}\right)^{b}\left(r_{2} \rho_{0}\right)^{c}\right\rangle
\end{aligned}
$$

where $a, b$ and $c$ are positive integers.

A toroidal edge-biregular map is fully regular if and only if one of the following is the case: $H=H_{\text {Rect }}$ and $a=c$; or $H=H_{\text {Rhomb }}$ and $b=c$. 
Proof Suppose $\left(H ; r_{0}, r_{2}, \rho_{0}, \rho_{2}\right)$ is a given edge-biregular map whose supporting surface is a torus. Thus, $H$ is necessarily finite, corresponding to a map of type $(4,4)$, and $H$ will have canonical partial presentation:

$$
H=\left\langle r_{0}, r_{2}, \rho_{0}, \rho_{2} \mid r_{0}^{2}, r_{2}^{2}, \rho_{0}^{2}, \rho_{2}^{2},\left(r_{0} r_{2}\right)^{2},\left(\rho_{0} \rho_{2}\right)^{2},\left(r_{0} \rho_{0}\right)^{2},\left(r_{2} \rho_{2}\right)^{2}, \ldots\right\rangle
$$

where the dots indicate some unknown extra relators which are yet to be determined. These necessary extra relators arise from $N_{T}$, the kernel of the epimorphism $\phi: \Delta \rightarrow$ $H$ where $\phi: R_{i} \rightarrow r_{i}$ and $\phi: P_{i} \rightarrow \rho_{i}$ for each $i \in\{0,2\}$; below, we determine all possibilities for the group $N_{T}$.

Since a map on the torus must be finite, each element of $H$ must have finite order. Specifically $r_{0} \rho_{2}$ must have finite order in $H$, let us say $a$. Thus, $\left(r_{0} \rho_{2}\right)^{a}$ is a relator in the group $H$ and since $a$ is, by definition, as small as possible, we may force $\left(r_{0} \rho_{2}\right)^{a}$ to be one of the two uniquely defined relators which are missing in the above partial presentation. Hence, $\left(R_{0} P_{2}\right)^{a} \in N_{T}$ and, by the minimality of $a$, we may assume it is one of a pair of generating elements in the rank two subgroup $N_{T} \leq T$. Geometric reasoning implies there must be another (independent, and also uniquely determined) translation generating $N_{T}$ which has the form $\left(R_{0} P_{2}\right)^{b}\left(R_{2} P_{0}\right)^{c}$ where $0 \leq b<a$ and $c>0$ is minimised.

The group $N_{T}=\left\langle\left(R_{0} P_{2}\right)^{a},\left(R_{0} P_{2}\right)^{b}\left(R_{2} P_{0}\right)^{c}\right\rangle$ is normal in the group $\Delta$ if and only if all conjugates of each of the generators of $N_{T}$ are themselves members of the subgroup $N_{T}$. It is clear that this is the case for the generator $\left(R_{0} P_{2}\right)^{a}$. It remains to consider conjugates of the element $\left(R_{0} P_{2}\right)^{b}\left(R_{2} P_{0}\right)^{c}$ in $\Delta$. We split this into two distinct cases. When $b=0$, all conjugates of $\left(R_{0} P_{2}\right)^{b}\left(R_{2} P_{0}\right)^{c}=\left(R_{2} P_{0}\right)^{c}$ are also within $N_{T}$, the lattice of equivalent points is rectangular, and $N_{T}=\left\langle\left(R_{0} P_{2}\right)^{a},\left(R_{2} P_{0}\right)^{c}\right\rangle$. This yields $H_{R e c t}$ in the statement of the Proposition. However, in the case when $b \neq 0$, then $N_{T}$ is normal if and only if we have both $\left(\left(R_{0} P_{2}\right)^{b}\left(R_{2} P_{0}\right)^{c}\right)^{R_{0}}=\left(R_{0} P_{2}\right)^{-b}\left(R_{2} P_{0}\right)^{c} \in$ $N_{T}$ or equivalently $\left(R_{0} P_{2}\right)^{2 b} \in N_{T}$, and also $\left(R_{2} P_{0}\right)^{2 c} \in N_{T}$. This implies $a \mid 2 b$ but $0<b<a$ so we must have $2 b=a$, meaning that the lattice of points, which is now equivalently generated by $N_{T}=\left\langle\left(R_{0} P_{2}\right)^{b}\left(R_{2} P_{0}\right)^{c},\left(R_{0} P_{2}\right)^{b}\left(R_{2} P_{0}\right)^{-c}\right\rangle$, is rhombic. This gives rise to the toroidal edge-biregular maps with presentations of the form $H_{\text {Rhomb }}$.

By construction, each of the above presentations $H_{\text {Rect }}$ and $H_{R h o m b}$ is uniquely determined from a given edge-biregular toroidal map which has an assigned alternateedge-colouring, and there can be no other toroidal proper edge-biregular maps.

These maps are fully regular if and only if the lattice is in fact a square lattice, that is, in the case of $H_{\text {Rect }}$ when $a=c$, and in the case of $H_{R h o m b}$ when $b=c$. Informally, this can be observed by looking at Fig. 12 and noting the need for all closed straight-ahead walks to have the same length in a fully regular map. More formally, the map is fully regular if and only if $\psi: r_{i} \leftrightarrow \rho_{i}$ for $i \in\{0,2\}$ is an automorphism, and inspection of the presentations of the groups quickly yields the same necessary and sufficient condition.

The reason for the use of the descriptors "rectangular" and "rhombic" is illustrated with an example of each of the corresponding maps shown in the diagrams in Fig. 12. 

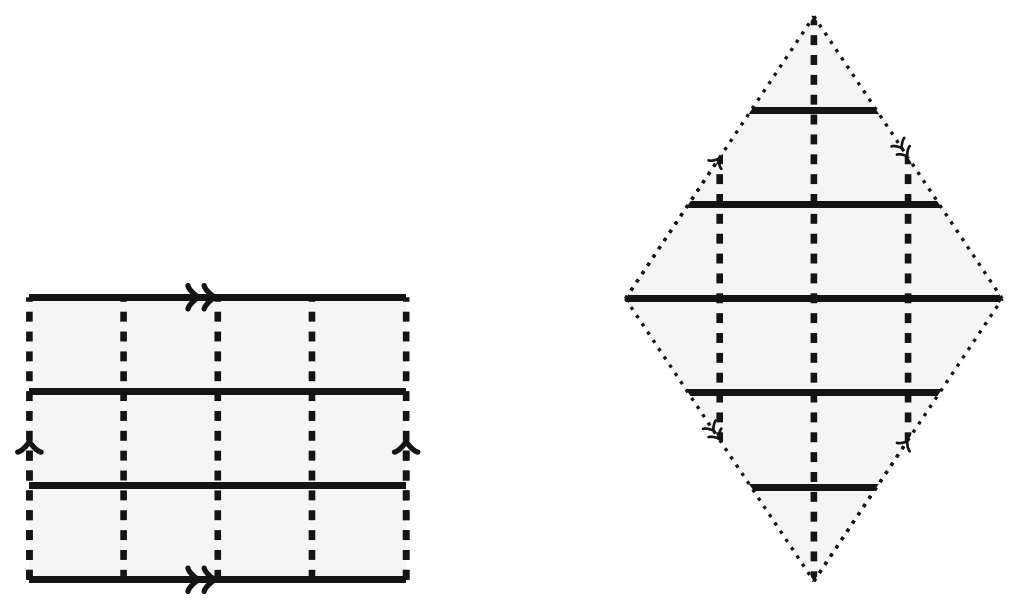

Fig. 12 Toroidal edge-biregular maps when $a=4, c=3$ : The lattice is rectangular, as shown on the left, when $b=0$, and rhombic when $b=2$

\subsubsection{Edge-biregular maps on the Klein bottle}

It is known that the Klein bottle supports no regular maps, so it follows that any edge-biregular map on this surface cannot have any semi-edges.

Since the Klein bottle is also Euclidean, an edge-biregular map on this surface is determined by forming the quotient of the infinite group $\Delta$ by a finite-index normal subgroup which in this case we denote $N_{K}$.

Proposition 4.4 Up to duality and twinness, edge-biregular maps on the Klein bottle are in one-to-one correspondence with groups $H$ with presentation:

$$
H=\left\langle r_{0}, r_{2}, \rho_{0}, \rho_{2} \mid r_{0}^{2}, r_{2}^{2}, \rho_{0}^{2}, \rho_{2}^{2},\left(r_{0} r_{2}\right)^{2},\left(\rho_{0} \rho_{2}\right)^{2},\left(r_{0} \rho_{0}\right)^{2},\left(r_{2} \rho_{2}\right)^{2},\left(r_{2} \rho_{0}\right)^{a} r_{0},\left(r_{0} \rho_{2}\right)^{b}\right\rangle
$$

where $a$ is a positive integer and $b \in\{1,2\}$.

Outline of proof The fundamental region for a Klein bottle is determined by a glide reflection and a translation in the direction perpendicular to the axis of reflection. See Coxeter and Moser, pp 40-43 in [4], or for further details of all uniform maps on this surface, see Wilson [16]. For an edge-biregular map, the grid must be mapped to itself by the glide reflection, maintaining the same colouring of edges. Thus, the axis of reflection must be orthogonal to the square grid and so, up to duality and twinness, we may assume the glide reflection is $\left(R_{2} P_{0}\right)^{a} R_{0}$ and the translation is $\left(R_{0} P_{2}\right)^{b}$ where $a$ and $b$ are positive integers and as small as possible.

Suppose $N_{K}=\left\langle\left(R_{2} P_{0}\right)^{a} R_{0},\left(R_{0} P_{2}\right)^{b}\right\rangle$ has finite index and is normal in $\Delta$. When this is the case, the quotient of $\Delta$ by $N_{K}$ will define an edge-biregular map on the Klein bottle. Conjugates of the translation $\left(R_{0} P_{2}\right)^{b}$ by elements of $\Delta$ are certainly in $N_{K}$, whereas $\left(\left(R_{2} P_{0}\right)^{a} R_{0}\right)^{P_{2}}=\left(R_{2} P_{0}\right)^{a} P_{2} R_{0} P_{2} \in N_{K}$ if and only if $\left(R_{0} P_{2}\right)^{2} \in N_{K}$, that is, if and only if $b \mid 2$. By an analogue of the proof of Proposition 4.3, for a given edge-biregular map on a Klein bottle, this yields the uniquely determined presentation for $H$ as given in the above Proposition, with the condition that $b \in 1,2$. 

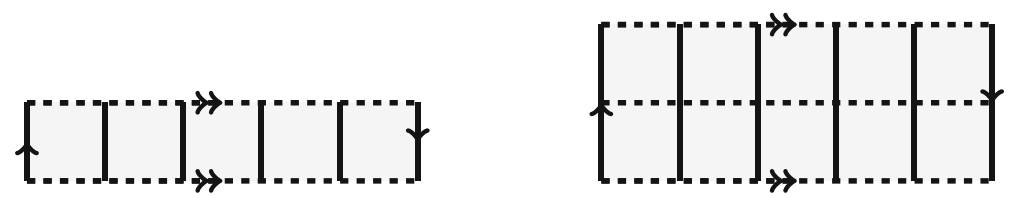

Fig. 13 Edge-biregular maps on the Klein bottle for $a=5$ where $b=1$ and $b=2$

Examples of these edge-biregular maps which are supported by the Klein bottle are shown in Fig. 13.

\subsubsection{Edge-biregular maps on the sphere and the projective plane}

We will consider edge-biregular maps on these surfaces according to the number of orbits of semi-edges.

As we have seen earlier, any semistar of even valency can be embedded in the sphere to form an edge-biregular map.

An edge-biregular map with exactly one semi-edge orbit must come from a fully regular map by construction 3 . It is well-known that the sphere supports fully regular maps of type $(3,3),(3,4),(4,3),(3,5),(5,3),(2, m)$ and $(m, 2)$ and so, by attaching a semi-edge at each corner, there are edge-biregular maps on the sphere of types $(6,6)$, $(6,8),(8,6),(6,10),(10,6),(4,2 m)$ and $(2 m, 4)$. Similarly the projective plane supports fully regular maps of type $(3,4),(4,3),(3,5),(5,3),(2, m)$ and $(m, 2)$ so on this surface there will be edge-biregular maps of types $(6,8),(8,6),(6,10),(10,6)$, $(4,2 m)$ and $(2 m, 4)$.

Finally, if an edge-biregular map contains no semi-edges, then (as now both the valency and the face length must be even) Euler's formula implies that on a sphere or a projective plane proper edge-biregular maps can only be embedded even cycles and their duals, of types $(2,2 m)$ and $(2 m, 2)$, respectively.

\section{Classification of proper edge-biregular maps when $H$ is dihedral and $\chi<0$}

A full classification of edge-biregular maps for surfaces of Euler characteristic $\chi=-p$ where $p$ is prime will appear in a forthcoming paper by Širán̆ and the author [11]. Here, we will conclude with a "dihedral classification". Henceforth, we will be focussing on surfaces with negative Euler characteristic and so we may assume that $k \geq 4$ and $l \geq 4$.

Dihedral groups can be generated by two involutions, so it seems natural that some of our groups $H$ will themselves be dihedral. The following working gives a classification, up to twinness and duality, of edge-biregular maps on surfaces for which $\chi<0$ when the group $H=\left\langle r_{0}, r_{2}, \rho_{0}, \rho_{2}\right\rangle \cong D_{2 m}$.

Since we know $V_{4} \cong\left\langle r_{0}, r_{2}\right\rangle \leq H$ (because there are no semi-edges), it is clear that $m$ is even. Therefore, $H$ has a central involution, which we will call $z$. We also know that $V_{4} \cong\left\langle\rho_{0}, \rho_{2}\right\rangle \leq H$. Having dealt with special cases in the previous section, 
an assumption we can now make is that the generators of $H$, namely $r_{0}, r_{2}, \rho_{0}$, and $\rho_{2}$, are distinct. For a dihedral group $D_{2 m}$ to have at least four distinct involutions, we must have $m \geq 4$, which forces the group to be non-abelian. Also, note that every copy of $V_{4}$ as a proper subgroup in a dihedral group contains the unique central involution $z$ and, taking account of the generators being distinct, this means $z \in\left\{r_{0} r_{2}, \rho_{0} \rho_{2}\right\}$.

By our choice of colouring for the edge orbits, that is up to twinness, we may assume $z=r_{0} r_{2}$. We also have $z \in\left\{\rho_{0}, \rho_{2}, \rho_{0} \rho_{2}\right\}$ which gives options which we address in turn.

\section{Suppose $z=\rho_{0} \rho_{2}$.}

In this case, $r_{0} r_{2}=\rho_{0} \rho_{2}$ and so $\rho_{0} r_{0}=\rho_{2} r_{2}$ which forces $k=l$. Also, $\rho_{2}=\rho_{0} r_{0} r_{2}$ so $H=\left\langle\rho_{0}, r_{0}, r_{2}\right\rangle=\left\langle\rho_{0}, r_{0}, r_{0} r_{2}\right\rangle$. But we know that $\left\langle\rho_{0}, r_{0}\right\rangle=D_{l}$ and so this leaves us with two possibilities:

Firstly, if $z=r_{0} r_{2} \in D_{l}$, then $H=D_{l}=\left\langle\rho_{0}, r_{0}\right\rangle \cong D_{2 m}$ and the type is $(2 m, 2 m)$. Notice, this map has just one face and one vertex and is supported by a surface with Euler characteristic $\chi=2-m$. By the fact the map has a single vertex, we also have $H=\left\langle r_{2}, \rho_{2}\right\rangle$;

Second, if $z=r_{0} r_{2} \notin D_{l}$, then, since $z$ is central, we get $H \cong D_{l} \times\langle z\rangle \cong D_{2 m}$. By the uniqueness of the central involutory element, this can only happen if $D_{l}$ has trivial centre, that is if the order of $\rho_{0} r_{0}$ is odd, which happens when $\frac{l}{2}=$ $\frac{m}{2}$ is odd. By considering the order of $\left(\rho_{0} r_{0}\right) z$, we see that $H=\left\langle\rho_{0}, r_{0}\right\rangle\langle z\rangle=$ $\left\langle\rho_{0}, r_{0} z\right\rangle=\left\langle\rho_{0}, r_{2}\right\rangle$. We also get, by remembering $\rho_{2}=\rho_{0} z$, that $H=\left\langle r_{0}, \rho_{2}\right\rangle$. These presentations yield a map of type $(m, m)$ which thus has two faces and two vertices, supported by a surface where $\chi=4-m$.

2. Now, let $z \in \rho_{0}, \rho_{2}$. Up to duality assume that $z=\rho_{0}$.

In this case, we have $r_{0} r_{2}=\rho_{0}$ so $r_{0} \rho_{0}=r_{2}$ which is an involution. Thus, $l=4$. Also, we get $H=\left\langle\rho_{2}, r_{2}, r_{0} r_{2}\right\rangle$. By similar reasoning to above, this gives us two possibilities:

Firstly, if $z \in D_{k}$, we have $H=D_{k}=\left\langle\rho_{2}, r_{2}\right\rangle \cong D_{2 m}$, the map has a single vertex with quadrangular faces, it is of type $(2 m, 4)$ and exists on a surface where $\chi=\frac{1}{2}(2-m)$;

Second, by the centrality of $z=r_{0} r_{2} \notin D_{k}$ we have $H=D_{k} \times\langle z\rangle=\left\langle r_{2}, \rho_{2}\right\rangle \times\langle z\rangle$. Also, by our assumption, $H \cong D_{2 m}$ and so the order of $r_{2} \rho_{2}$ must be odd and $H=\left\langle z r_{2}, \rho_{2}\right\rangle=\left\langle r_{0}, \rho_{2}\right\rangle$. The map is of type $(m, 4)$ where $\frac{m}{2}$ is odd, and thus, the map has two vertices. This map occurs on a surface with $\chi=\frac{1}{2}(4-m)$.

Note that these (non-orientable) maps are not regular since regularity would require an automorphism of the group $H$ which swaps $\rho_{0}$, which is central in $H$, with $r_{0}$ which is not central. 
These results can be tabulated as follows giving a classification, up to duality and twinness, of edge-biregular maps on surfaces for which $\chi<0$ when the group $H$ is dihedral, $H=\left\langle r_{0}, r_{2}, \rho_{0}, \rho_{2}\right\rangle \cong D_{2 m}$ where $m$ is even and $m \geq 4$. For completeness, we note that dihedral edge-biregular maps on the sphere, projective plane, torus and Klein bottle also exist, and they are included in our earlier analysis.

\begin{tabular}{|c|c|c|c|c|c|c|c|}
\hline Type & $H \cong D_{2 m}$ & Relations & Conditions & $\chi<0$ & V & $F$ & Regular \\
\hline$(2 m, 2 m)$ & $\begin{array}{l}\left\langle r_{0}, \rho_{0}\right\rangle \\
\left\langle r_{2}, \rho_{2}\right\rangle\end{array}$ & $r_{0} r_{2}=\rho_{0} \rho_{2}=\left(r_{0} \rho_{0}\right)^{\frac{m}{2}}=\left(r_{2} \rho_{2}\right)^{\frac{m}{2}}$ & None & $2-m$ & 1 & 1 & Yes \\
\hline$(m, m)$ & $\begin{array}{l}\left\langle r_{2}, \rho_{0}\right\rangle \\
\left\langle r_{0}, \rho_{2}\right\rangle\end{array}$ & $r_{0} r_{2}=\rho_{0} \rho_{2}=\left(r_{2} \rho_{0}\right)^{\frac{m}{2}}=\left(r_{0} \rho_{2}\right)^{\frac{m}{2}}$ & $\frac{m}{2}$ is odd & $4-m$ & 2 & 2 & Yes \\
\hline$(2 m, 4)$ & $\left\langle r_{2}, \rho_{2}\right\rangle$ & $r_{0} r_{2}=\rho_{0}=\left(r_{2} \rho_{2}\right)^{\frac{m}{2}}$ & None & $\frac{1}{2}(2-m)$ & 1 & $\frac{m}{2}$ & No \\
\hline$(m, 4)$ & $\left\langle r_{0}, \rho_{2}\right\rangle$ & $r_{0} r_{2}=\rho_{0}=\left(r_{0} \rho_{2}\right)^{\frac{m}{2}}$ & $\frac{m}{2}$ is odd & $\frac{1}{2}(4-m)$ & 2 & $\frac{m}{2}$ & No \\
\hline
\end{tabular}

Remark 5.1 It is worth noting that this classification, along with the earlier work, ensures that there is no closed surface which does not support edge-biregular maps. Specifically, all non-orientable surfaces with negative Euler characteristics support an edge-biregular map of type $(4(1-\chi), 4)$, as well as its dual map of type $(4,4(1-$ $\chi)$ ), and their twins, while the orientable surface with negative Euler characteristic $\chi$ supports an edge-biregular map of type $(2(2-\chi), 2(2-\chi))$.

\section{Concluding remarks}

This paper presents the background of edge-biregular maps and addresses the existence of these maps on surfaces with boundary components and surfaces with non-negative Euler characteristic as well as, for other closed surfaces, a classification for edgebiregular maps with dihedral colour-preserving automorphism groups.

Edge-biregular maps on surfaces of negative prime Euler characteristic are classified in an upcoming paper by Širáň and the author [11]. Further research into edge-biregular maps is planned, and the author also hopes to investigate the lesser-studied types of maps which arise from other index two subgroups of the full triangle groups.

Acknowledgements The author wishes to thank Jozef Širán̆ for many helpful and enjoyable discussions during the preparation of this paper. The author would also like to thank the anonymous referee whose careful reading of the original manuscript and resulting suggestions are much appreciated.

Funding Not applicable.

Data availability Not applicable as the results presented in this manuscript rely on no external sources of data or code.

\section{Declarations}

Conflict of interest The author declares no conflict of interest that are relevant to the content of this article. 
Open Access This article is licensed under a Creative Commons Attribution 4.0 International License, which permits use, sharing, adaptation, distribution and reproduction in any medium or format, as long as you give appropriate credit to the original author(s) and the source, provide a link to the Creative Commons licence, and indicate if changes were made. The images or other third party material in this article are included in the article's Creative Commons licence, unless indicated otherwise in a credit line to the material. If material is not included in the article's Creative Commons licence and your intended use is not permitted by statutory regulation or exceeds the permitted use, you will need to obtain permission directly from the copyright holder. To view a copy of this licence, visit http://creativecommons.org/licenses/by/4.0/.

\section{References}

1. Archdeacon, D., Bonnington, C.P., Širáň, J.: Regular pinched maps. Australas. J. Combin. 58(1), 16-26 (2014)

2. Archdeacon, D., Gvozdjak, P., Širáň, J.: Constructing and forbidding automorphisms in lifted maps. Math. Slovaca 47(2), 113-129 (1997)

3. Bryant, R.P., Singerman, D.: Foundations of the theory of maps on surfaces with boundary. Q. J. Math. 2(36), 17-41 (1985)

4. Coxeter, H.S.M., Moser, W.O.J.: Generators and Relations for Discrete Groups, 4th edn. Springer, Berlin (1980)

5. d'Azevedo, B., Catalano, D.A., Širáň, J.: Bi-rotary maps of negative prime characteristic. Ann. Comb. 23(1), 27-50 (2019)

6. Duarte, R.: 2-Restrictedly-regular hypermaps of small genus. Doctoral thesis, University of Aveiro, Portugal (2007)

7. Graver, J.E., Watkins, M.E.: Locally Finite, Planar, Edge-Transitive Graphs, vol. 26, No. 601. Memoirs of the American Mathematical Society (1997)

8. Hubard, I., Orbanić, A., Weiss, A.I.: Monodromy groups and self-invariance. Canad. J. Math. 61(6), 1300-1324 (2009)

9. Jones, G.A.: Edge-transitive maps. arXiv:1605.09461v3 [math.CO] (2019)

10. Jones, G.A., Singerman, D.: Theory of maps on orientable surfaces. Proc. Lond. Math. Soc. 3(37), 273-307 (1978)

11. Jeans, O., Širáň, J.: Classifying edge-biregular maps of negative prime Euler characteristic. In press with the Art of Discrete and Applied Mathematics. https://doi.org/10.26493/2590-9770.1392.f9a

12. Orbanić, A., Pellicer, D., Weiss, A.I.: Map operations and $k$-orbit maps. J. Combin. Theory Ser. A 117, 411-429 (2010)

13. Širáň, J.: How symmetric can maps on surfaces be? In: Blackburn, S.R., Gerke, S., Wildon, M. (eds.) Surveys in Combinatorics 2013. London Mathematical Society Lecture Note Series 409, pp. 161-238. Cambridge University Press, Cambridge (2013)

14. Wilson, S.E.: Riemann surfaces over regular maps. Canad. J. Math 30(4), 763-782 (1978)

15. Wilson, S.E.: Edge-transitive maps and non-orientable surfaces. Math. Slovaca 47, 65-83 (1997)

16. Wilson, S.: Uniform maps on the Klein bottle. J. Geom. Graph. 10(2), 161-171 (2006)

Publisher's Note Springer Nature remains neutral with regard to jurisdictional claims in published maps and institutional affiliations. 